\title{
ON RECURRENCE FOR SELF-SIMILAR ADDITIVE PROCESSES
}

\author{
KOUJI YAMAMURO
}

\section{Introduction}

In our paper a stochastic process is called an additive process if it is a stochastically continuous process with independent increments and has rightcontinuous sample functions with left limits a.s. Self-similar additive processes constitute an important class of additive processes which are not assumed to be time-homogeneous. But they have not been studied except in some papers, e.g. [2], [3], [4], and [5]. We investigated their transience and recurrence in [3] and [5]. The dichotomy of recurrence and transience for this class of processes is known (see [3]). But a criterion for recurrence and transience has not been found. As an important example, there is a strictly stable process on $\boldsymbol{R}^{d}$, which is a self-similar Lévy process, that is, a self-similar time-homogeneous additive process. It is recurrent if its index $\alpha$ satisfies $\max \{1, d\} \leq \alpha \leq 2$, where its exponent is $\alpha^{-1}$ if we regard it as a self-similar additive process (see the definition below). So we attempted to find a new method to prove recurrence for strictly stable processes with index $\max \{1, d\} \leq \alpha \leq 2$ without using time-homogeneity. We succeeded in our attempt and we could find recurrence conditions showing great differences between self-similar additive processes and Lévy processes. We note that this problem cannot be solved by using the existing methods because of the difficulty caused by the fact that the expected occupation times on open sets containing 0 cannot determine recurrence (see [3]).

A self-similar additive process is defined by the following.

Definition. A stochastic process $\left\{X_{t}: t \geq 0\right\}$ on $\boldsymbol{R}^{d}$, which is defined on a probability space $(\Omega, \mathscr{F}, P)$, is called a self-similar additive process, or a process of class $L$, with exponent $H>0$ if it satisfies the following conditions:

(i) $\left\{X_{c t}\right\}$ and $\left\{c^{H} X_{t}\right\}$ have the same finite-dimensional distributions for every $c>0$,

(ii) $X_{t_{1}}-X_{t_{0}}, X_{t_{2}}-X_{t_{1}}, \ldots, X_{t_{n}}-X_{t_{n-1}}$ are independent for any $n$ and any choice of $0 \leq t_{0}<t_{1}<t_{2}<\cdots<t_{n}$

(iii) almost surely $X_{t}$ is right-continuous in $t \geq 0$ and has left limits in $t>0$.

Throughout this paper let $\left\{X_{t}\right\}$ be a self-similar additive process on $\boldsymbol{R}^{d}$ with

Received April 26, 1999; revised October 6, 1999. 
exponent $H$. Note that it is stochastically continuous and $X_{0}=0$ a.s. Let $D=$ $\left\{x \in \boldsymbol{R}^{d}:|x| \leq 1\right\}, S=\left\{x \in \boldsymbol{R}^{d}:|x|=1\right\}$, and $1_{D}(x)$ be the indicator function of $D$. Then $\left\{X_{t}\right\}$ has the following characteristic function:

$$
\begin{aligned}
E e^{i\left\langle z, X_{t}\right\rangle}= & E e^{l\left\langle t^{H} z, X_{1}\right\rangle} \\
= & \exp \left[-t^{2 H} 2^{-1}\langle A z, z\rangle+i t^{H}\langle\gamma, z\rangle\right. \\
& \left.+\int_{S} \sigma(d \xi) \int_{0}^{\infty}\left(e^{i t^{H}\langle z, r \xi\rangle}-1-i t^{H}\langle z, r \xi\rangle 1_{D}(r \xi)\right) \frac{k_{\xi}(r)}{r} d r\right]
\end{aligned}
$$

for $z \in \boldsymbol{R}^{d}$, where $A$ is a symmetric and nonnegative matrix, $\gamma \in \boldsymbol{R}^{d}, \sigma$ is a probability measure on $S, k_{\xi}(r)$ is nonnegative, nonincreasing right continuous in $r$ and Borel measurable in $\xi$, and

$$
\int_{S} \sigma(d \xi) \int_{0}^{\infty} \min \left\{r, r^{-1}\right\} k_{\xi}(r) d r<\infty .
$$

For any random variable $Z$ we denote by $P_{Z}$ the distribution of $Z$, and by $\hat{P}_{Z}(z)$ the characteristic function of $P_{Z}$. We have the following main results.

THEOREM 1.1. Let $d=1$. The process $\left\{X_{t}\right\}$ is recurrent if it satisfies one of the following:

(i) $A \neq 0$,

(ii) $k(r)-\varphi(1 / r)$ is nonnegative and nonincreasing on $(0, \varepsilon)$ for some $\varepsilon>0$, where $k(r)=k_{+1}(r)+k_{-1}(r)$ and $\varphi(r)$ is a nonnegative strictly increasing convex function on $(1 / \varepsilon, \infty)$.

THeOREM 1.2. Let $d=2$. If the rank of the matrix $A$ is 2 , then the process $\left\{X_{t}\right\}$ is recurrent.

Remark. When $d=2$, there is a transient self-similar additive process such that the matrix $A$ has rank 1. For example, $\left\{X_{t}\right\}$ is transient if $X_{1}$ is full and has the following characteristic function: Suppose that

$$
\begin{gathered}
\int_{S} \sigma(d \xi) k_{\xi}(0+)<\infty . \\
\hat{P}_{X_{1}}(z)=\exp \left[-2^{-1}\langle A z, z\rangle+i\left\langle\gamma_{0}, z\right\rangle+\int_{S} \sigma(d \xi) \int_{0}^{\infty}\left(e^{l\langle z, r \xi\rangle}-1\right) \frac{k_{\xi}(r)}{r} d r\right],
\end{gathered}
$$

where $\gamma_{0}=\left(\begin{array}{l}\gamma_{01} \\ \gamma_{02}\end{array}\right), c_{1}, c_{2} \in \boldsymbol{R}$, and $A=\left(\begin{array}{ll}c_{1} \gamma_{01} & c_{2} \gamma_{01} \\ c_{1} \gamma_{02} & c_{2} \gamma_{02}\end{array}\right)$. In fact $\left\{\Pi R X_{t}\right\}$ is transient according to Theorem 1.1 in [5], where the $1 \times 2$-matrix $\Pi$ is equal to (1 0 ) and $R$ is a rotation matrix such that the first coordinate of $R \gamma_{0}$ is 0 .

Our theorems show differences between a self-similar addititve process and a Lévy process, that is, when the two processes have the same distribution at time 
1 , there is a case where one of them is recurrent and the other is transient. For example, let $\left\{B_{t}\right\}$ be a $d$-dimensional Brownian motion and let $\gamma \neq 0$. If $d \leq 2$, then $\left\{B_{t}+\sqrt{t} \gamma\right\}$, which is a self-similar additive process with exponent $2^{-1}$, is recurrent by the above theorems. On the other hand we know that the Lévy process $\left\{B_{t}+t \gamma\right\}$ is transient (see [1]).

\section{Proofs}

Let

$$
f(x)=\prod_{j=1}^{d} \max \left\{1-\left|x_{j}\right|, 0\right\} .
$$

Then

$$
\int_{\boldsymbol{R}^{d}} f(x) d x=1
$$

and the Fourier transform of $f$ is

$$
\hat{f}(z)=\int_{\boldsymbol{R}^{d}} e^{l\langle z, x\rangle} f(x) d x=\prod_{J=1}^{d}\left(\frac{\sin 2^{-1} z_{J}}{2^{-1} z_{J}}\right)^{2} .
$$

We note that

$$
f(x)=\frac{1}{(2 \pi)^{d}} \int_{\boldsymbol{R}^{d}} e^{-\imath\langle z, x\rangle} \hat{f}(z) d z .
$$

Define

$$
a_{n}=\sum_{k=1}^{n} k^{-H d}, \quad W_{n}=a_{n}^{-1} \sum_{k=1}^{n} f\left(X_{k}\right) .
$$

We will show two facts:

$$
\begin{gathered}
\lim _{n \rightarrow \infty} E W_{n}>0, \\
\sup _{n} E\left[W_{n}^{2}\right]<\infty,
\end{gathered}
$$

for some $H$ with $0<H d \leq 1$. Then the recurrence of $\left\{X_{t}\right\}$ is shown in the following way. As (2.2) implies the uniform integrability of $\left\{W_{n}\right\}$, we have

$$
E\left[\limsup _{n \rightarrow \infty} W_{n}\right] \geq \lim _{n \rightarrow \infty} E W_{n}>0 .
$$

Hence we have

$$
P\left(\lim _{n \rightarrow \infty} \sum_{k=1}^{n} f\left(X_{k}\right)=\infty\right)>0
$$

and $\left\{X_{t}\right\}$ is not transient. Therefore it is recurrent by Theorem 3.2 in [3]. The proof of (2.1) and (2.2) will be complete in Lemma 2.3. 
LEMMA 2.1. Let $0<\beta \leq 1$.

(i) If $\gamma>\beta>0$, then we have $\lim _{n \rightarrow \infty} \sum_{J=1}^{n} j^{-\gamma}\left(\sum_{J=1}^{n} j^{-\beta}\right)^{-1}=0$.

(ii) We have $\sup _{n \geq 2} \sum_{1 \leq J_{1}<J_{2} \leq n} j_{1}^{-\beta}\left(j_{2}-j_{1}\right)^{-\beta}\left(\sum_{J=1}^{n} j^{-\beta}\right)^{-2}<\infty$.

Proof is omitted.

LEMMA 2.2. Let $H d \leq 1$. If

then we have

$$
\int_{\boldsymbol{R}^{d}}|z|\left|\hat{P}_{X_{1}}(z)\right| d z<\infty
$$

$$
\lim _{n \rightarrow \infty} E W_{n}=\frac{1}{(2 \pi)^{d}} \int_{R^{d}} \hat{P}_{X_{1}}(z) d z>0 .
$$

Proof. Let $c_{0}=(2 \pi)^{-d} \int_{\boldsymbol{R}^{d}} \hat{P}_{X_{1}}(z) d z$. We have $c_{0}>0$. Indeed, the support of $P_{X_{1}}$ is $\boldsymbol{R}^{1}$, because $\int_{0}^{1} k(r) d r \geq \int_{0}^{1} \varphi(1 / r) d r=\infty$ and it is shown from the general theory of infinitely divisible distributions (see [1]) under the assumption of Theorem 1.1(ii), and because $P_{X_{1}}$ is the convolution of a Gaussian distribution and some distribution under the other conditions. Hence, since $P_{X_{1}}$ is unimodal by Yamazato's theorem [6], its density is positive on $\boldsymbol{R}^{1}$.

We have

$$
\left|E W_{n}-c_{0}\right| \leq \frac{1}{\sum_{k=1}^{n} k^{-H d}} \sum_{k=1}^{n} k^{-H d}\left|k^{H d} E f\left(X_{k}\right)-c_{0}\right|=J_{n}, \quad \text { (say). }
$$

Since

$$
\begin{aligned}
k^{H d} E f\left(X_{k}\right) & =k^{H d} \int_{\boldsymbol{R}^{d}} f(x) d x \frac{1}{(2 \pi)^{d}} \int_{\boldsymbol{R}^{d}} e^{-\imath\langle z, x\rangle} \hat{\boldsymbol{P}}_{X_{1}}\left(k^{H} z\right) d z \\
& =\frac{1}{(2 \pi)^{d}} \int_{\boldsymbol{R}^{d}} f(x) d x \int_{\boldsymbol{R}^{d}} e^{-\imath\left\langle z, k^{-H} x\right\rangle} \hat{\boldsymbol{P}}_{X_{1}}(z) d z
\end{aligned}
$$

we obtain that

$$
\begin{aligned}
J_{n} & \leq \frac{1}{\sum_{k=1}^{n} k^{-H d}} \sum_{k=1}^{n} \frac{1}{k^{H d}(2 \pi)^{d}} \int_{\boldsymbol{R}^{d}} f(x) d x \int_{\boldsymbol{R}^{d}}\left|e^{-\iota\left\langle z, k^{-H} x\right\rangle}-1\right|\left|\hat{P}_{X_{1}}(z)\right| d z \\
& \leq \frac{1}{\sum_{k=1}^{n} k^{-H d}} \sum_{k=1}^{n} \frac{1}{k^{H d}(2 \pi)^{d}} \int_{\boldsymbol{R}^{d}} f(x) d x \int_{\boldsymbol{R}^{d}}|z| k^{-H}|x|\left|\hat{P}_{X_{1}}(z)\right| d z \\
& =\text { const. } \times \sum_{k=1}^{n} k^{-H(d+1)}\left(\sum_{k=1}^{n} k^{-H d}\right)^{-1}
\end{aligned}
$$

Hence, from Lemma 2.1(i), we have $\lim _{n \rightarrow \infty} J_{n}=0$. 
LEMMA 2.3. If $H=2^{-1}$ under the assumption of Theorem 1.1(i) or Theorem 1.2, or if $H=1$ under the assumption of Theorem 1.1(ii), then we have (2.1) and (2.2).

Proof. Under the assumption of the lemma we have $\int_{R^{d}}|z|\left|\hat{P}_{X_{1}}(z)\right| d z<\infty$, which is proved even under the assumption of Theorem 1.1(ii) in the same way as in the proof of the inequality (2.3) shown later. Hence we obtain (2.1) from Lemma 2.2 .

Let $j_{2}>j_{1}$. Using the Fourier inverse transformation of $f$, we have

$$
\begin{aligned}
E f\left(X_{J_{1}}\right) f\left(X_{J_{2}}\right) & =E\left[f\left(X_{J_{1}}\right) E\left[f\left(X_{J_{2}}-X_{J_{1}}+x\right)\right]_{x=X_{J_{1}}}\right] \\
& =\frac{1}{(2 \pi)^{2 d}} \int_{\boldsymbol{R}^{d}} \hat{f}(z) \hat{P}_{X_{J_{2}}-X_{J_{1}}}(z) d z \int_{\boldsymbol{R}^{d}} \hat{f}(y) \hat{P}_{X_{J_{1}}}(y+z) d y \\
& \leq \frac{1}{j_{1}^{H d}(2 \pi)^{2 d}} \int_{\boldsymbol{R}^{d}}\left|\hat{P}_{X_{J_{2}}-X_{J_{1}}}(z)\right| d z \int_{\boldsymbol{R}^{d}}\left|\hat{P}_{X_{1}}(y)\right| d y .
\end{aligned}
$$

Here we have used self-similarity.

Therefore, in order to show (2.2), it suffices to prove boundedness of

$$
I_{n}=\sum_{J_{1}=1}^{n} E f^{2}\left(X_{J_{1}}\right)\left(\sum_{J_{1}=1}^{n} j_{1}^{-H d}\right)^{-2}
$$

and

$$
J_{n}=\sum_{1 \leq J_{1}<J_{2} \leq n} j_{1}^{-H d} \int_{R^{d}}\left|\hat{P}_{X_{J_{2}}-X_{J_{1}}}(z)\right| d z\left(\sum_{J_{1}=1}^{n} j_{1}^{-H d}\right)^{-2} .
$$

Since $0 \leq f \leq 1$, we have $\lim \sup _{n \rightarrow \infty} I_{n}=0$ in view of Lemma 2.2. Hence $\sup _{n} I_{n}<\infty$.

Now we shall show boundedness of $J_{n}$. First we shall consider the case that the rank of matrix $A$ is $d$. Let $H=2^{-1}$. We have

$$
\begin{aligned}
J_{n} & \leq \sum_{1 \leq J_{1}<J_{2} \leq n} j_{1}^{-2^{-1} d} \int_{R^{d}} e^{-2^{-1}\left(J_{2}-J_{1}\right)\langle A z, z\rangle} d z\left(\sum_{J_{1}=1}^{n} j_{1}^{-2^{-1} d}\right)^{-2} \\
& =\sum_{1 \leq J_{1}<J_{2} \leq n} \frac{1}{j_{1}^{2^{-1} d}\left(j_{2}-j_{1}\right)^{2^{-1} d}} \int_{R^{d}} e^{-2^{-1}\langle A y, y\rangle} d y\left(\sum_{J_{1}=1}^{n} j_{1}^{-2^{-1} d}\right)^{-2} .
\end{aligned}
$$

Hence, from Lemma 2.1(ii), we have $\sup _{n} J_{n}<\infty$.

Next we shall consider the case of Theorem 1.1(ii). It suffices only to consider the case that $A=0$. Let $H=1$. Now we have

$$
\sum_{1 \leq J_{1}<J_{2} \leq n} \frac{1}{j_{1}} \int_{\boldsymbol{R}^{1}}\left|\hat{P}_{X_{J_{2}}-X_{J_{1}}}(z)\right| d z \leq \sum_{1 \leq J_{1}<J_{2} \leq n} \frac{1}{j_{1}^{2}} \int_{\boldsymbol{R}^{1}}\left|\hat{P}_{X_{J_{2} / J_{1}}-X_{1}}(z)\right| d z .
$$


We divide the last integral into two parts according as $|z| \leq 1$ or $|z|>1$. Suppose that $|z| \leq 1$. We have

$$
\left|\hat{P}_{X_{1}}(z)\right|^{-1}=\exp \left[\int_{0}^{\infty}(1-\cos (z r)) \frac{k(r)}{r} d r\right] \leq C_{1} .
$$

Here the constant $C_{1}$ does not depend on $z$. Since $\varphi(r) \geq c_{1} r-c_{2}$ on $\left(1 / \varepsilon_{1}, \infty\right)$ for some constants $c_{1}>0, c_{2} \in \boldsymbol{R}^{1}$, and $0<\varepsilon_{1}<\varepsilon$, then we have $k(r)>c_{3} / r$ on $\left(0, \varepsilon_{2}\right)$ for some constants $c_{3}>0$ and $0<\varepsilon_{2}<\varepsilon_{1}$. Hence we obtain that

$$
\begin{aligned}
\left|\hat{P}_{X_{J_{2} / j_{1}}}(z)\right| & =\exp \left[\int_{0}^{\infty}(\cos (z r)-1) \frac{k\left(\left(j_{1} / j_{2}\right) r\right)}{r} d r\right] \\
& \leq \exp \left[\int_{0}^{\varepsilon_{2}\left(J_{2} / j_{1}\right)}(\cos (z r)-1) \frac{c_{3}}{r^{2}} \frac{j_{2}}{j_{1}} d r\right] \\
& =\exp \left[c_{3} \frac{j_{2}|z|}{j_{1}} \int_{0}^{\left(J_{2} / j_{1}\right) \varepsilon_{2}|z|}(\cos u-1) \frac{d u}{u^{2}}\right] .
\end{aligned}
$$

Since $\left|\hat{P}_{X_{J_{2} / j_{1}}-X_{1}}(z)\right|=\left|\hat{P}_{X_{J_{2} / 1_{1}}}(z)\right| /\left|\hat{P}_{X_{1}}(z)\right|$ and $|z| \int_{0}^{\varepsilon_{2}|z|}(1-\cos u) u^{-2} d u \sim K|z|$ as $|z| \rightarrow \infty$ with some positive constant $K$, we obtain that

$$
\begin{aligned}
& \sum_{1 \leq J_{1}<J_{2} \leq n} \frac{1}{j_{1}^{2}} \int_{|z| \leq 1}\left|\hat{P}_{X_{J_{2} / j_{1}}-X_{1}}(z)\right| d z \\
& \quad \leq \sum_{1 \leq J_{1}<J_{2} \leq n} \frac{C_{1}}{j_{1} j_{2}} \int_{|z| \leq J_{2} / j_{1}} \exp \left[c_{3}|z| \int_{0}^{\varepsilon_{2}|z|}(\cos u-1) \frac{d u}{u^{2}}\right] d z \\
& \quad \leq \text { const. } \times \sum_{1 \leq J_{1}<J_{2} \leq n} \frac{1}{j_{1} j_{2}} .
\end{aligned}
$$

Next Suppose that $|z|>1$. Then, since $\varphi\left(j_{2} /\left(j_{1} r\right)\right)-\varphi(1 / r) \geq c_{4}\left(j_{2} / j_{1}-1\right) r^{-1}$ on $\left(0, \varepsilon_{3}\right)$ for some constants $c_{4}>0$ and $0<\varepsilon_{3}<\varepsilon$, letting $\delta=\min \left\{\varepsilon_{3}, 1\right\}$, we have

$$
\begin{aligned}
\left|\hat{P}_{X_{J_{2} / J_{1}}-X_{1}}(z)\right| & =\exp \left[\int_{0}^{\infty}(\cos (z r)-1) \frac{k\left(\left(j_{1} / j_{2}\right) r\right)-k(r)}{r} d r\right] \\
& \leq \exp \left[c_{4} \int_{0}^{\delta /|z|}(\cos (z r)-1)\left(\frac{j_{2}}{j_{1}}-1\right) \frac{1}{r^{2}} d r\right] \\
& \leq \exp \left[-K_{1}|z|^{2} \frac{j_{2}-j_{1}}{j_{1}} \int_{0}^{\delta /|z|} d r\right] \\
& =\exp \left[-K_{2}|z| \frac{j_{2}-j_{1}}{j_{1}}\right]
\end{aligned}
$$


with some positive constants $K_{1}$ and $K_{2}$. Hence we have

$$
\begin{aligned}
& \sum_{1 \leq J_{1}<J_{2} \leq n} \frac{1}{j_{1}^{2}} \int_{|z| \geq 1}\left|\hat{P}_{X_{J_{2} / J_{1}}-X_{1}}(z)\right| d z \\
& \quad \leq \sum_{1 \leq J_{1}<J_{2} \leq n} \frac{1}{j_{1}^{2}} \int_{|z| \geq 1} \exp \left[-K_{2}|z| \frac{j_{2}-j_{1}}{j_{1}}\right] d z \\
& \quad \leq \sum_{1 \leq J_{1}<J_{2} \leq n} \frac{1}{j_{1}\left(j_{2}-j_{1}\right)} \int_{R^{1}} e^{-K_{2}|z|} d z .
\end{aligned}
$$

Hence, from Lemma 2.1(ii), we have $\sup _{n} J_{n}<\infty$. This completes the proof of Lemma 2.3.

Example 2.4. Let $\left\{X_{t}\right\}$ be strictly stable with index $\alpha$ satisfying $1 \leq \alpha<2$. Letting $\varphi(r)=c r^{\alpha}$ for some $c>0$, we obtain that $\left\{X_{t}\right\}$ is recurrent from Theorem 1.1(ii).

The following example is pointed out by K. Sato.

Example 2.5. Let $\varphi(r)=c r^{\alpha}(\log r)^{\beta}$, where three constants $c, \alpha$, and $\beta$ satisfy one of the following conditions:

(i) $c>0,1<\alpha<2$, and $\beta \in \boldsymbol{R}^{1}$,

(ii) $c>0, \alpha=1$, and $\beta \geq 0$,

(iii) $c>0, \alpha=2$, and $\beta<-1$.

Then, if $k(r)=\varphi(1 / r)$ on $(0, \varepsilon)$ for small enough $\varepsilon$, then $k(r)$ satisfies the condition of Theorem 1.1(ii).

Acknowledgements. The author thanks Professor K. Sato for his valuable comments. The formulation of the condition in Theorem 1.1(ii) is suggested by him. The author's original condition was that $\sigma(\{+1\})>0$ and $k_{+1}(r)-c r^{-1}$ is nonegative and nonincreasing on $(0, \varepsilon)$ for some $\varepsilon>0$ and $c>0$. He also thanks the refree for helpful comments.

\section{REFERENCES}

[1] K. SATo, Processes with Independent Increments, Kinokuniya, 1990 (in Japanese).

[2] K. Sato, Self-similar processes with independent increments, Probab. Theory Related Fields, 89 (1991), 285-300.

[3] K. Sato and K. Yamamuro, On selfsımilar and semi-selfsimilar processes with independent increments, J. Korean Math. Soc., 35 (1998), 207-224.

[4] T. Watanabe, Sample function behavior of increasing processes of class $L$, Probab. Theory Related Fields, 104 (1996), 349-374.

[5] K. Yamamuro, Transience conditions for self-similar additive processes, in J. Math. Soc. Japan, 52 (2000), 343-362. 
[6] M. Yamazato, Unımodality of infintely divisible distribution functions of class $L$, Ann. Probab., 6 (1978), 523-531.

\section{Aichi Konan College}

TAKAYA-CHO, KONAN 483-8086

JAPAN 